\title{
An efficient algorithm for the Closest String Problem
}

\author{
Omar Vilca ${ }^{1}$, Rosiane de Freitas ${ }^{1}$ \\ ${ }^{1}$ Institute of Computation - Federal University of Amazonas (UFAM) \\ Manaus, AM - Brasil \\ \{omarlatorre, rosiane\}@icomp.ufam.edu.br
}

\begin{abstract}
The closest string problem that arises in computational molecular biology and coding theory is to find a string that minimizes the maximum Hamming distance from a given set of strings, the CSP is NP-hard problem. This article proposes an efficient algorithm for this problem with three strings. The key idea is to apply normalization for the CSP instance. This enables us to decompose the problem in five different cases corresponding to each position of the strings. Furthermore, an optimal solution can be easily obtained in linear time. A formal proof of the algorithm will be presented, also numerical experiments will show the effectiveness of the proposed algorithm.
\end{abstract}

\section{Introduction}

String selection problems are among the most important topics facing researchers in computational biology. Combinatorial optimization is a possible approach to solving selection sequences problems. There are some previously exact algorithms for CSP with 3-sequences [Gramm et al. 2001, Liu et al. 2011], in both papers presented algorithms in linear time. Meneses obtains optimal solutions via integer programming [Meneses et al. 2004, Meneses et al. 2005], In [Vilca 2013] solves this problem by cutting planes algorithm.

The CSP is defined as follows: Given a finite set $S=\left\{s^{1}, s^{2}, \ldots, s^{n}\right\}$ of strings with alphabet $\Sigma$, each string with length $m$, find a center string $t$ of length $m$ minimizing $d$, such that for every string $s^{i} \in S, d_{H}\left(t, s^{i}\right) \leq d$, we mean the Hamming distance between $t$ and $s^{i}$. This is a NP-hard problem according to Frances and Litman[Frances and Litman 1997]. In the following, we show a formal proof of correctness from an efficient linear time algorithm for CSP instances with 3-sequences, computational experiments are reported, and finally, concluding remarks are presented.

\section{Efficient linear time algorithm for 3-sequences}

The 3-CSP-A algorithm is designed based on the isomorphic instance [Gramm et al. 2001]. An exact algorithm for 3-CSP with alphabet $\Sigma=2$ is found in [Liu et al. 2011].

Definition 1 (Normalized instance) Let $S$ an instance, that is, $S=\left\{s^{1}, \ldots, s^{n}\right\}$, where $\left|s^{i}\right|=m$, with $1 \leq i \leq n$ and $1 \leq j \leq m$. Let $M_{n \times m}$ a matrix of characters from $S$ each 
column is a position of the strings in $S$, so we have $M\left[c_{1}, c_{2}, \ldots, c_{m}\right]$

$$
\phi\left(c_{j}^{\prime}\right)_{1 \leq i \leq n, 1 \leq j \leq m}= \begin{cases}\lambda_{1} & \text { if } \sigma_{1} \in c_{j}: \max _{\sigma_{i}}\left|c_{j}^{i}=\sigma_{1}\right| \\ \lambda_{2} & \text { else if } \sigma_{2} \in c_{j}: \max _{\sigma_{i}}\left|c_{j}^{i} \backslash \sigma_{1}=\sigma_{2}\right| \\ \ldots & \\ \lambda_{k} & \text { else if } \sigma_{k} \in c_{j}: \max _{\sigma_{i}}\left|c_{j}^{i} \backslash\left\{\sigma_{1}, \ldots, \sigma_{k-1}\right\}=\sigma_{k}\right|\end{cases}
$$

Let $M^{\prime}\left[c_{1}^{\prime}, \ldots, c_{m}^{\prime}\right]$, and $S^{\prime}$ an instance based on characters from $M^{\prime}$, as a result $S^{\prime}$ is called normalized instance from $S$.

Theorem 1 Let a CSP instance with 3-sequences, which denotes 3-CSP, that is, $S=$ $\left\{s^{i} \in \Sigma^{m}, i=1,2,3\right\}$ with alphabet $|\Sigma|>2$, so the 3-CSP-A algorithm always finds an exact solution to 3-CSP.

Proof 1 Let $S=\left\{s^{1}, s^{2}, s^{3}\right\}$ a 3-CSP instance, let $\phi$ a bijective function that transforms $S$ into $S^{\prime}$, called normalized instance that is equivalent to $S$ for $\phi$ according to [Gramm et al. 2001]. Let $M_{3 \times m}$ a matrix of characters from $S$ each column is a position of the strings in $S$, so we have $M\left[c_{1}, c_{2}, \ldots, c_{m}\right]$ with $m$ length of strings in $S$

$$
\phi\left(c_{j}\right)_{1 \leq i \leq 3,1 \leq j \leq m}= \begin{cases}a & \text { if } \sigma_{1} \in c_{j}: \max _{\sigma_{i}}\left|c_{j}^{i}=\sigma_{1}\right| \\ b & \text { else if } \sigma_{2} \in c_{j}: \max _{\sigma_{i}}\left|c_{j}^{i} \backslash \sigma_{1}=\sigma_{2}\right| \\ c & \text { else if } \sigma_{3} \in c_{j}: \max _{\sigma_{i}}\left|c_{j}^{i} \backslash\left\{\sigma_{1}, \sigma_{2}\right\}=\sigma_{3}\right|\end{cases}
$$

We assume wlog five different cases in $c_{i}$, that is, $v_{1}=a a a, v_{2}=b a a, v_{3}=a b a$, $v_{4}=a a b$ and $v_{5}=a b c$. In order to get an optimal solution, divide $M^{\prime}\left[c_{1}, c_{2}, \ldots, c_{m}\right]$ in blocks of 3-length and 2-length, consider all possible combinations of $\left\{v_{2}, \ldots, v_{5}\right\}$, then drop $v_{1}$ since it is a trivial case.

In the case of 3-length blocks with repetition columns we have, $d_{H}\left(v_{i} v_{j} v_{k}, b a a\right)=$ 2 and without repetitions $d_{H}\left(v_{i} v_{j} v_{k}, a a a\right)=1$ for $2 \leq i, j, k \leq 4$ now inserting $v_{5}$ in our analysis with column repetition $d_{H}\left(\left\{v_{5} v_{i} v_{j}, v_{i} v_{5} v_{j}, v_{i} v_{j} v_{5}\right\}, a a a\right)=2$, and finally $d_{H}\left(v_{5} v_{5} v_{5}, a b c\right)=2$.

In the case of 2-length blocks we have, $d_{H}\left(v_{i} v_{j}, a a\right)=1$ and $d_{H}\left(v_{i} v_{i}, a b\right)=1$ with $2 \leq i, j \leq 4$ after add $v_{5}, d_{H}\left(v_{2} v_{5}, a a\right)=1, d_{H}\left(v_{3} v_{5}, a b\right)=1, d_{H}\left(v_{4} v_{5}, a c\right)=1$, and finally $d_{H}\left(v_{5} v_{5}, a a\right)=2$.

So in truth we are interested in the cases when the Hamming distance is equals to 1 . Let $l_{j}$ a number that accounts for each time the case $v_{j}$ is repeated where $j \in$ $\{1,2, \ldots, 5\}$, let $t^{\prime}$ a string of size $m$, an optimal solution of $S^{\prime}$, in order to minimize the maximum Hamming distance $d_{H}\left(t^{\prime}, S^{\prime}\right)$, we verified various reductions for each case $v_{j}$ presented. We are checking each kind of reductions for 2-length and 3-length blocks.

Assume wlog $l_{2} \leq l_{3} \leq l_{4}$, then $l_{3}=l_{3}-l_{2}, l_{4}=l_{4}-\left(l_{2}+l_{3}\right)$, after these calculations, one of them is greater or equal to zero. let $\rho_{\text {iab }}=\left\lfloor\frac{1}{2} l_{i}\right\rfloor$ for $2 \leq i \leq 4$

- If $l_{5} \bmod 3>0$ and $\left\{l_{2}, l_{3}, l_{4}\right\} \bmod 2>0$ then $\left\{\rho_{5 a c}, \rho_{a b}, \rho_{a a}\right\}=1$

- If $l_{5} \bmod 2>0$ then $\rho_{5 a b}=1$

Let $t$ a string that represents an optimal solution of 3-CSP-A, with $1 \leq i \leq m$, we 
have:

$$
\begin{array}{lllll}
s_{i}^{1}=s_{i}^{2} & & & & t_{i}=s_{i}^{1}=s_{i}^{2} \\
s_{i}^{1}=s_{i}^{2} & s^{2} \neq s^{3} & & \rho_{2 a b}>0 & t_{i}=s_{i}^{3} \\
s_{i}^{1} \neq s_{i}^{2} & s_{i}^{1}=s_{i}^{3} & & \rho_{3 a b}>0 & t_{i}=s_{i}^{2} \\
s_{i}^{1} \neq s_{i}^{2} & s_{i}^{1} \neq s_{i}^{3} & s_{i}^{2}=s_{i}^{3} & \rho_{4 a b}>0 & t_{i}=s_{i}^{1} \\
s_{i}^{1} \neq s_{i}^{2} & s_{i}^{2} \neq s_{i}^{3} & s_{i}^{2} \neq s_{i}^{3} & \rho_{5 a b}>0 & t_{i}=s_{i}^{2} \\
s_{i}^{1} \neq s_{i}^{2} & s_{i}^{2} \neq s_{i}^{3} & s_{i}^{2} \neq s_{i}^{3} & \rho_{5 a c}>0 & t_{i}=s_{i}^{3} \\
s_{i}^{1} \neq s_{i}^{2} & s_{i}^{2} \neq s_{i}^{3} & s_{i}^{2} \neq s_{i}^{3} & \rho_{a b}>0 & t_{i}=s_{i}^{2} \\
s_{i}^{1} \neq s_{i}^{2} & s_{i}^{2} \neq s_{i}^{3} & s_{i}^{2} \neq s_{i}^{3} & \rho_{a a}>0 & t_{i}=s_{i}^{1}
\end{array}
$$

Thus the theorem holds.

Example 1 Let $S_{1}$ a 3-CSP instance, and let $S_{2}$ its normalized instance, we have:

$$
S_{1}=\left\{\begin{array}{l}
A G T A T T G G T G \\
C C C T T T G A G A \\
T A G T G G G T C T
\end{array}\right.
$$

$$
S_{2}=\left\{\begin{array}{l}
\text { aaabaaaaaa } \\
\text { bbbaaaabbb } \\
\text { cccabbaccc }
\end{array}\right.
$$

After using the 3-CSP-A algorithm we have an optimal solution for the normalized instance $S_{2}$, that is, $t=$ caccaaaaaa, with Hamming distance $d_{H}\left(t, S_{2}\right)=4$.

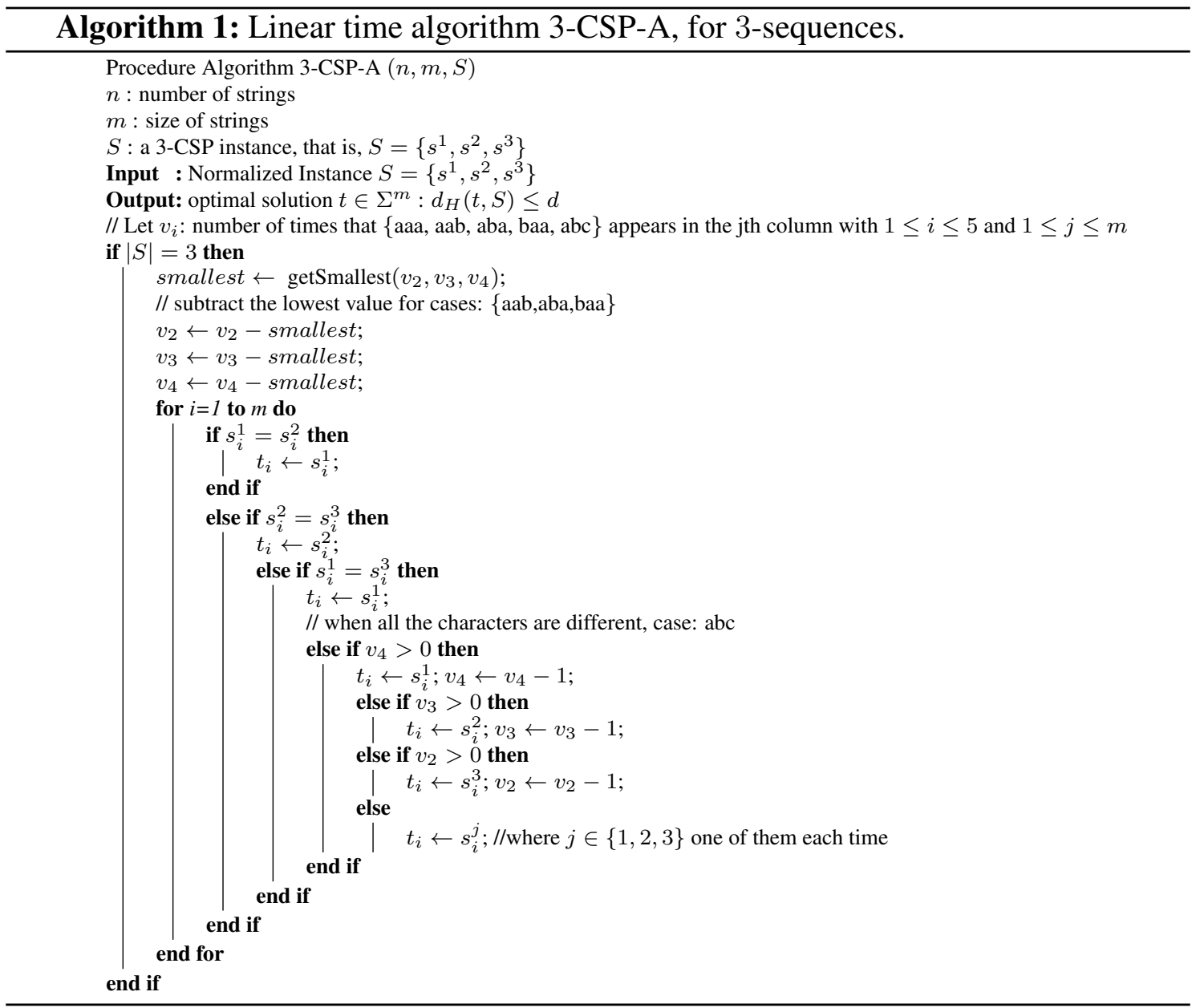




\begin{tabular}{l|llc|lcc|lcc}
\hline Instance & \multicolumn{3}{|c|}{ 2 Characters } & \multicolumn{3}{c|}{ 4 Characters } & \multicolumn{3}{c}{ 20 Characters } \\
\hline $\mathrm{m}$ & seed & Val & time(s) & seed & Val & time(s) & seed & Val & time(s) \\
\hline 100 & 542319 & 27 & $<1$ & 791034 & 48 & $<1$ & 691425 & 63 & $<1$ \\
200 & 7121 & 52 & $<1$ & 52151 & 91 & $<1$ & 351554 & 124 & $<1$ \\
300 & 64874 & 77 & $<1$ & 68724 & 132 & $<1$ & 98121 & 183 & $<1$ \\
400 & 6487 & 112 & $<1$ & 193185 & 180 & $<1$ & 246754 & 244 & $<1$ \\
500 & 94115 & 124 & $<1$ & 15364 & 215 & $<1$ & 658745 & 311 & $<1$ \\
600 & 5419 & 153 & $<1$ & 5419 & 260 & $<1$ & 154525 & 373 & $<1$ \\
700 & 43212 & 180 & $<1$ & 524514 & 306 & $<1$ & 487754 & 437 & $<1$ \\
800 & 2454 & 215 & $<1$ & 55364 & 354 & $<1$ & 754812 & 494 & $<1$ \\
900 & 645387 & 234 & $<1$ & 6487 & 389 & $<1$ & 722451 & 557 & $<1$ \\
1000 & 94315 & 260 & $<1$ & 153364 & 444 & $<1$ & 34567 & 616 & $<1$ \\
2000 & 264554 & 508 & $<1$ & 6487 & 881 & 1 & 65743 & 1219 & 1 \\
3000 & 68174 & 765 & 2 & 2454 & 1305 & 2 & 4432 & 1857 & 1 \\
4000 & 4212 & 1003 & 3 & 53214 & 1760 & 3 & 543 & 2484 & 3 \\
5000 & 722312 & 1278 & 5 & 934145 & 2188 & 6 & 344 & 3099 & 5 \\
\hline \hline
\end{tabular}

Table 1. Linear time algorithm 3-CSP-A, for 3-sequences.

\section{Computational Experiments and Results}

Some computational experiments was done involving forty two 3-CSP instances, using the instance generator described in the literature [Meneses et al. 2004]. These instances consider alphabets with two, four and twenty characters, which obtained optimal solutions using the 3-CSP-A algorithm presented in the Section 2. The header on Table 2 has the following meanings: the first column indicates the tested instance, indicating the parameters $(m)$ size of string; the columns $(2,4$, and 20 Characters) indicates the (seed) number to generate a random instance, (Val) values of optimal solutions to 3 -sequences for binary, DNA, and protein types as well as their execution times in seconds.

\section{Final Remarks}

We proposed an exact algorithm for the special case of CSP with 3-sequences and alphabet size $|\Sigma|>2$, given the corresponding theoretical analysis.

\section{References}

Frances, M. and Litman, A. (1997). On covering problems of codes. Theory of Computing Systems, 30(2):113-119.

Gramm, J., Niedermeier, R., and Rossmanith, P. (2001). Exact solutions for closest string and related problems. In Proceedings of the 12th International Symposium on Algorithms and Computation, ISAAC '01, pages 441-453.

Liu, X., Liu, S., Hao, Z., and Mauch, H. (2011). Exact algorithm and heuristic for the closest string problem. Computers \& Operations Research, 38(11):1513-1520.

Meneses, C., Lu, Z., Oliveira, C., and Pardalos, P. (2004). Optimal solutions for the closest string problem via integer programming. INFORMS Journal on Computing, 16(4):419-429.

Meneses, C., Oliveira, C., and Pardalos, P. (2005). Optimization techniques for string selection and comparison problems in genomics. Engineering in Medicine and Biology Magazine, IEEE, 24(3):81-87.

Vilca, O. L. (2013). Métodos para problemas de seleção de cadeias de caracteres. Master's thesis, Universidade Federal do ABC, Santo André, São Paulo. 